\title{
Wall shear stress measurements by white-light oil-film interferometry
}

\author{
J. Lunte ${ }^{1} \mathbb{D} \cdot$ E. Schülein ${ }^{1}$
}

Received: 22 October 2019 / Accepted: 11 February 2020 / Published online: 2 March 2020

(c) The Author(s) 2020

\begin{abstract}
Wall shear stress and wall streamline direction measurements were conducted on a generic delta wing model to demonstrate the potential of the white-light oil-film interferometry in three-dimensional flows. With the proposed algorithm, an automatic oil-film thickness calculation is possible and yields reproducible and plausible wall shear stress distributions on the leeward side of the delta wing for various angles of attack.
\end{abstract}

\section{Graphic abstract}

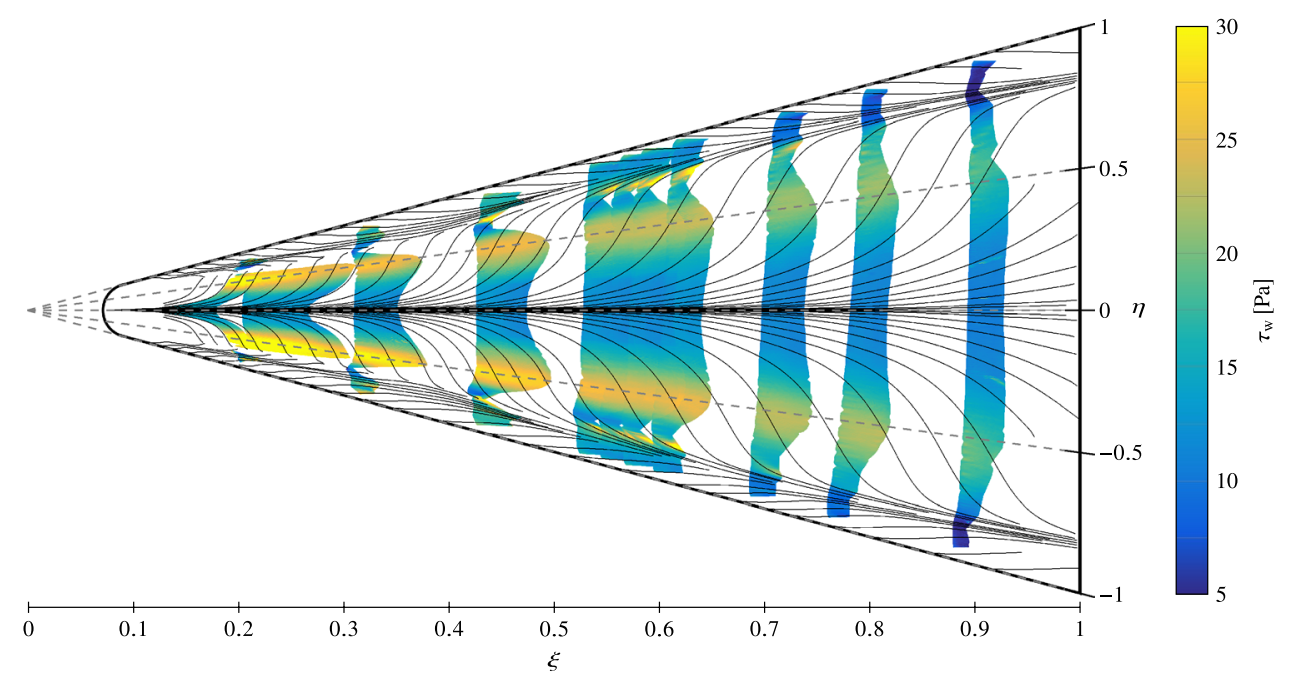

\section{List of symbols}

$\alpha \quad$ Angle of attack

C Constant

$c_{r} \quad$ Chord length

$\delta$ Delta function

$\Delta s_{\text {opt }} \quad$ Optical path difference

$\eta \quad$ Dimensionless spanwise coordinate

$h \quad$ Oil-film thickness

I Intensity

$I_{0} \quad$ Mean intensity

$I_{\mathrm{A}} \quad$ Amplitude of the intensity oscillation

J. Lunte

jens.lunte@dlr.de

1 German Aerospace Center (DLR), Institute of Aerodynamics and Flow Technology, Bunsenstr. 10, 37073 Göttingen, Germany
$L_{\text {c }} \quad$ Coherence length

$\lambda \quad$ Wave length

$\lambda_{0} \quad$ Mean wave length

$\mu_{\text {oil }} \quad$ Oil viscosity

$n \quad$ Stream tube width

$n_{\text {air }} \quad$ Refractive index of the air

$n_{\text {eff }} \quad$ Effective refractive index

$n_{\text {oil }} \quad$ Refractive index of the oil

$R \quad$ Nose radius of the model

$R_{1} \quad$ Reattachment line of the primary vortex

$R_{2} \quad$ Reattachment line of the secondary vortex

$S \quad$ Spanwise distance to the leading edge

$s \quad$ Streamline coordinate

$s^{\prime} \quad$ Scaled streamline coordinate

$s_{\text {max }} \quad$ Streamline length

$S_{2} \quad$ Separation line of the secondary vortex 
$t \quad$ Time

$\tau_{\mathrm{w}} \quad$ Wall shear stress

$\theta_{\mathrm{i}} \quad$ Angle of incident of the light

$x \quad$ Longitudinal coordinate

$\xi \quad$ Dimensionless longitudinal coordinate

$y \quad$ Spanwise coordinate

\section{Introduction}

Knowledge of wall shear stress (WSS) distributions is of major interest in the aerodynamics, because they indicate the topology of the wall-bounded flow, the position of separation bubbles as well as the location of laminar-turbulent boundary layer transition. The WSS is also an important validation property for CFD simulations, because it is determined through the flow close to the wall and therefore sensitive to the simulated boundary layer. Opposing the informative benefits of the WSS are the challenges in measuring this flow quantity. Over the past decades, various skin friction measurement techniques were developed, improved and tested. Detailed overviews of these techniques are given by Winter (1979), Settles (1986), Haritonidis (1989) and Naughton and Sheplak (2002).

The oil-film interferometry (OFI) has become widely used as a skin friction measurement technique as it yields accurate results with low equipment cost. The foundation for the OFI was given by Squire (1962) in 1962, who theoretically showed that the motion of a thin oil-film is solely defined through the shear stress. The objective of Squire was to attest that the oil flow coincides with the wall streamline direction. Tanner and Blows (1976) inverted the relation given by Squire and measured the development of the oilfilm thickness through interferometry to calculate the WSS.

The necessary interferometry data to calculate the oilfilm thickness can be measured either as a spatial interferometry pattern through an image or by recording the time history of the interference pattern at a fixed point as the oil-film becomes thinner. In the beginning of the OFI technique, spatial interferometry patterns were seldom measured (Tanner and Kulkarni 1976; Tanner and Blows 1976), because the images were taken on film and therefore difficult to analyze. Instead, single-point measurements with a focused laser beam were conducted and the interference signal was recorded with a photo-detector (Kim and Settles 1990; Monson and Higuchi 1981; Tanner 1977). This single-point technique became known under the name laser interferometry skin friction measurements (LISF). The LISF technique provided accurate results even in complex flows (Monson 1983) and was also applied in three-dimensional (3-D) flows (Kim and Settles 1990; Monson 1984). But, the LISF method is tedious as soon as the number of measurement points increases.
Since the use of CCD cameras became widespread, images of spatial interferometry patterns were measured and analyzed more frequently. Spatial interferometry patterns include the necessary information to calculate the skin friction at multiple points during a single run and greatly surpass LISF techniques in this point. Over the decades, different approaches were presented to calculate the skin friction from the spatial interferometry patterns, which can be classified by the number of utilized interference images. The single-image algorithms (Garrison and Ackman 1998; Mateer et al. 1996; Monson et al. 1993) have the advantage that they require no optical access to the measuring section as the interference images can be taken after wind-tunnel shutdown. On the opposite, they are receptive for start-up and shutdown effects of the wind tunnel and also require the exact duration of the measurement. The downsides of the single-image method can be avoided by using multiimage methods (Driver and Drake 2008), but they require an optical access to the measurement section. The modern trend goes to further improve the accuracy of the dual-image methods by using multiple images of spatial interference patterns (Naughton and Hind 2013).

Despite over four decades of development since the beginning of the OFI, WSS measurements in 3-D flows are still seldom performed (Driver 1997; Kim and Settles 1990; Monson 1984; Naughton and Brown 1997; Schülein 2006; Zilliac 1998), and the results are usually presented only at cross sections or specific points. The reasons for the absent of experimental global skin friction distributions are the requirements on the measurement. The calculation of a surface WSS distribution requires the information of the wall streamline directions and also the temporal development of the oil-film thickness distribution. Although the interference pattern contains the complete information of the oil-film thickness distribution, the function for mapping the oil-film thickness to the interference signal is not bijective, which makes the inverse determination of the oil-film thickness challenging.

The absence of an inverse function led to a diversity of algorithms, and nearly every author developed his own heuristic approach to determine the oil-film thickness. Most algorithms use only the extremes of the interference pattern (Driver and Drake 2008; Mateer et al. 1996; Monson and Higuchi 1981; Naughton and Hind 2013) and therefore neglect nearly the complete information that the whole interference pattern could provide. Other common simplifications are the smoothing of the interference pattern (Kim and Settles 1990; Mateer et al. 1996; Naughton and Brown 1996; Siller et al. 1992) or the assumption of a locally constant WSS (Kim and Settles 1990; Monson and Higuchi 1981; Naughton and Hind 2013), which implies a linear slope in the oil-film thickness. All these algorithms perform well for their designed test cases, but also make 
it obvious that the OFI still lacks a generally applicable algorithm for the measurement of global skin friction distributions.

This paper provides a physically based automatic algorithm, which calculates the temporal development of the oil-film thickness pixelwise from a sequence of interference patterns. The algorithm combines the accuracy and robustness of the LISF techniques with the spatial distribution provided by the image techniques.

The measurement of the local wall streamline direction becomes increasingly complex with the demands on the WSS measurement. While the wall streamline direction is obvious in 2D flows and the pointwise skin friction measurements just need the local streamline direction, the calculation of surface WSS distributions requires the information of the streamline direction in the whole measurement region. The wall streamline direction of the entire measurement region can be determined by hand, but reaching the necessary resolution to justify linear interpolation is cumbersome. Baldwin et al. (2019) recently published surface skin friction distributions for a 3-D flow field generated by a fin at Mach two. For the determination of the surface streamlines, Baldwin et al. applied a cross-correlation based algorithm, initially designed for PIV analysis, on a sequence of surface oil flow images for an automatic calculation of the wall streamline direction. Although such algorithms provide a wall streamline distribution in a fraction of the time, the results have to be assessed carefully until such methods are validated thoroughly. In this paper, a heuristic algorithm will be presented for interpolating additional wall streamlines from a limited set of hand-drawn lines. The presented interpolation technique surpasses common interpolation methods, which are based on the nearest neighboring points, by using the curve characteristics of the hand-drawn wall streamlines (e.g., turning points) to enable useful interpolation over wide regions. The presented interpolation method requires only a reduced number of hand-drawn lines and still yields the necessary accuracy to calculate global skin friction distributions.

OFI measurements on a generic delta wing will be used to explain and demonstrate our algorithms for the determination of the oil-film thickness and wall streamline direction. The obtained information about the oil-film thickness and wall streamline directions will be used to calculate surface WSS distributions over the delta wing for different angles of attack. The flow topology of the delta wing provides a suitable test case to show the qualities as well as the limitations of the presented methods.

Parts of this work were presented in the scope of the STAB conference (Lunte and Schülein 2018) and the AIAA Aviation Forum 2018 (Lunte et al. 2018).

\section{Optical skin friction measurements}

The wall shear stress measurement with the oil-film interferometry is based on the mass conservation law and was first demonstrated by Tanner and Blows (1976). The surface of the model is covered with an oil-film exposed to the flow. The mass flow of a transparent oil-film element, like it is shown in Fig. 1a, is bounded between both limiting streamlines and can only flow through the cross section A.

For thin oil-films, this mass flow through the cross section A can be described as a Couette flow (Naughton and Sheplak 2002). This yields the following relationship between the thinning of the oil-film upstream of A and the wall shear stress, which was presented by Maksimov et al. (1994):

$\tau_{w}=-\frac{2 \mu_{\mathrm{oil}}}{n_{A} h_{A}^{2}} \frac{\mathrm{d}}{\mathrm{d} t} \int_{0}^{s_{A}} n h \mathrm{~d} s$

with the oil viscosity $\mu_{\text {oil }}$, the time derivative $\mathrm{d} / \mathrm{d} t$, the width of the oil stream tube $n$, the distance along the streamline $s$, and the oil-film thickness $h$. The parameters $s_{A}$ and $h_{A}$ represent the values at the actual cross section $\mathrm{A}$.

The thinning of the oil-film is measured via interferometry, which is shown in Fig. 1b. An incident light beam is

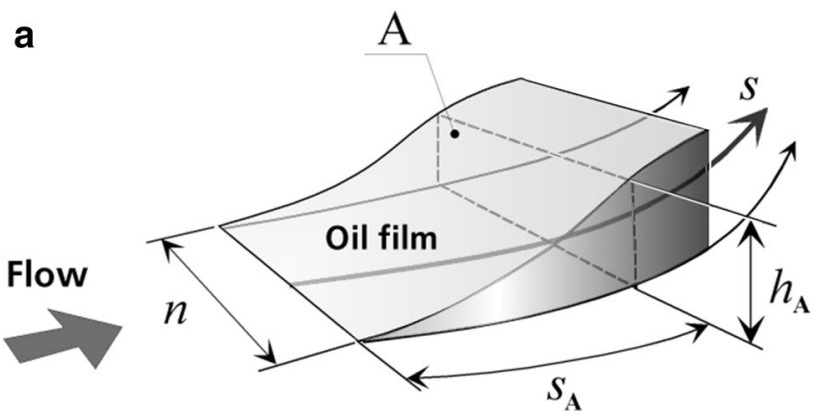

b
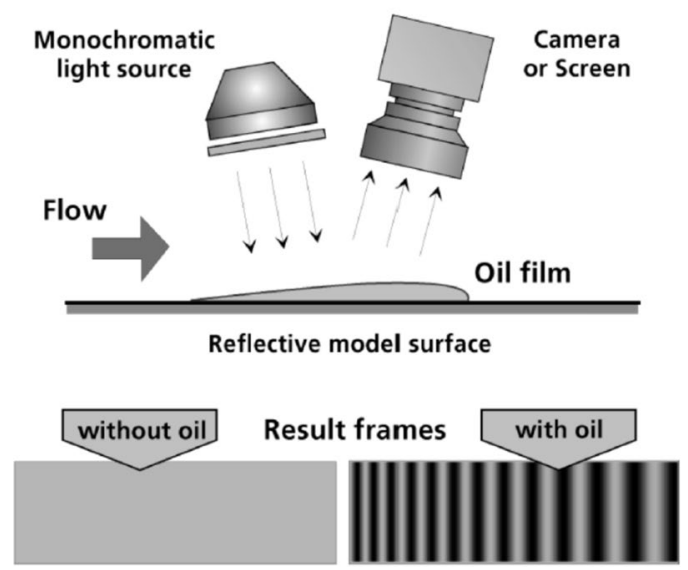

Fig. 1 a Oil-film element. b Principle of oil-film interferometry (Schülein 2006) 
partially reflected at the oil-film and at the model surface. Therefore, the optical path difference $\Delta s_{\text {opt }}$ depends on the oil-film height and leads to an interference pattern. Interference measurements are mostly conducted with monochromatic light but also a white light source can be used (Desse 2003). White light has the drawback of a short coherence length, which leads to less visible interference fringes, but enhances the calculation accuracy due to the multiple wave lengths involved. A customary digital color camera is able to record three different wavelength ranges (color channels) due to the Bayer filter on the chip. Assuming a Gaussian spectral density distribution for each color channel (red, green, blue), the measured intensity $I$ of each channel is given by Lunte (2016):

$I\left(\Delta s_{\mathrm{opt}}\right)=I_{0}+I_{\mathrm{A}} \exp \left[-\left(\frac{\Delta s_{\mathrm{opt}}}{L_{\mathrm{c}}}\right)^{2}\right] \cos \left(2 \pi \frac{\Delta s_{\mathrm{opt}}}{\lambda_{0}}\right)$.

Here, $I_{0}$ and $I_{\mathrm{A}}$ represent the mean light intensity and the amplitude of its maximum oscillation, $\lambda_{0}$ is the mean wave length, and $L_{\mathrm{c}}$ the coherence length of the selected color channel. The optical path difference of the interferometer is given by

$\Delta s_{\text {opt }}=2 h \sqrt{n_{\text {oil }}^{2}-n_{\text {air }}^{2} \sin ^{2} \theta_{\mathrm{i}}}=2 h n_{\mathrm{eff}}$,

where $n_{\text {oil }}$ and $n_{\text {air }}$ are the refractive indices of oil and air. The angle of incidence of the light is given by $\theta_{\mathrm{i}}$, and an effective refractive index $n_{\text {eff }}$ is introduced for a better overview of the equations. Figure 2 shows schematically the relation between Eq. 2 and the oil-film thickness $h$.

\section{Experimental setup}

The test object is a delta wing with a flat upper side and sharp leading edges, as shown in Fig. 3. It has a sweep angle of $75^{\circ}$, a span of $150 \mathrm{~mm}$, and a chord of $c_{r}=260.2 \mathrm{~mm}$. The nose of the wing is rounded in the $\mathrm{x}, \mathrm{y}$-plane $(R=6.9 \mathrm{~mm})$,

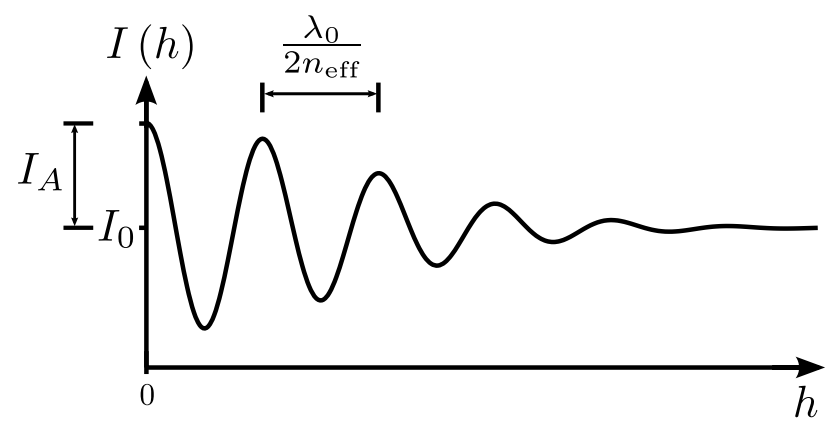

Fig. 2 Interference intensity for a single color channel depending on the oil-film thickness $h$

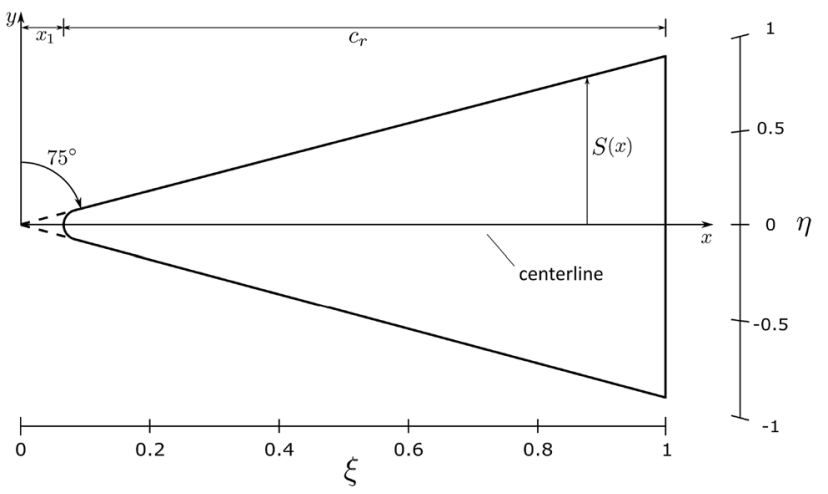

Fig. 3 Geometry of the delta wing

and the length of the wing from the virtual wing peak to the trailing edge is $280 \mathrm{~mm}$. The established dimensionless coordinates (Hummel 2004) $\xi=x /\left(c_{r}+x_{1}\right)$ (longitudinal coordinate) and $\eta=y / S(x)$ (spanwise coordinate) are used for the description of the model.

The low speed wind tunnel $1 \mathrm{MG}$ at DLR Göttingen was used for the experiments. The 1MG is continuously operated Göttingen-type wind tunnel with an open test section of a $1 \mathrm{~m} \times 0.7 \mathrm{~m}$ cross section. In the present study, the freestream velocity was $50.22 \mathrm{~m} / \mathrm{s}$, which leads to a Reynolds number of $R e=0.8 \times 10^{6}$ based on the chord. The model was investigated at various angles of attack between $5^{\circ}$ and $25^{\circ}$.

The optical setup consists of a white-light LED-panel $(600 \mathrm{~mm} \times 600 \mathrm{~mm})$ and a customary digital color camera Sony NEX-6. Prior to the experiments, the spectrum of the LED-panel was measured by a spectrometer to obtain the mean wavelengths. The surface of the model was coated with a reflective PVC-foil to increase the visibility of the interference pattern. Transparent ELBESIL silicon oil with a nominal kinematic viscosity of $v=100 \mathrm{~mm}^{2} / \mathrm{s}$ was used. It was applied to the surface before the start of the wind tunnel as a narrow strip across the longitudinal axis of the model. During the wind-tunnel runs, interference images were acquired every $5 \mathrm{~s}$ until approximately 40 images were obtained. After each run, the temperature of the model surface was measured to take account of the resulting oil viscosity variation.

\section{Reconstruction of interference images}

The reconstruction of the interference images is performed in a series of steps, which are automated in the developed postprocessing tool. From a sequence of interference images, the used algorithm can calculate the oil-film thickness at each point utilizing the oil-film equation (Squire 1962). The thin oil-film equation describes the relationship 
between the temporal development of the oil-film thickness $h$ and the occurring wall shear stress. At a fixed location on the wall and under steady flow conditions, the values $\tau_{w}, \mu_{\text {oil }}$ and $n$ remain constant over time. Under these conditions, the thin oil-film equation can be simplified and the temporal development of $h$ at each position is given by $h=C \cdot t^{-1}$ (Brown and Naughton 1999), where $t$ is the effective time from the start of the flow (see Ref. Tanner and Blows 1976 for details) and $C$ is a proportionality constant. The constant $C$ varies with the location on the model surface. Inserting the expression $h=C \cdot t^{-1}$ into Eq. (2) yields the temporal development of the measured light intensity at a fixed location:

$$
I(t)=I_{0}+I_{\mathrm{A}} \underbrace{\exp \left[-\left(\frac{2 n_{\mathrm{eff}} C}{L_{\mathrm{c}} t}\right)^{2}\right]}_{f\left(t^{-1}\right)} \underbrace{\cos \left(2 \pi \frac{2 n_{\mathrm{eff}} C}{\lambda_{0} t}\right)}_{g\left(t^{-1}\right)} .
$$

Figure 4a shows the measured intensity for the three color channels of the camera for one pixel over the time. In Fig. 4b, these intensities are plotted against the reciprocal time $t^{-1}$. The shape of the curves is now similar to the function in Fig. 2. A Fourier transformation of the data is performed in order to compute the constant $C$ in Eq. 4 for each pixel. With the convolution theorem, the Fourier transform of Eq. (4) can be written as

$\mathcal{F}(I)=\mathcal{F}\left(I_{0}\right)+I_{\mathrm{A}} \mathcal{F}(f \cdot g)=\mathcal{F}\left(I_{0}\right)+I_{\mathrm{A}} \mathcal{F}(f) * \mathcal{F}(g)$.
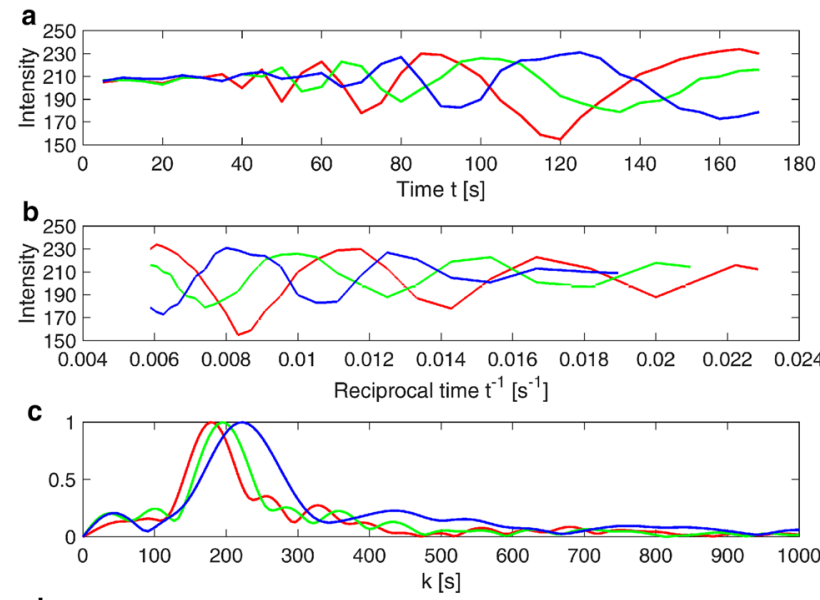

d

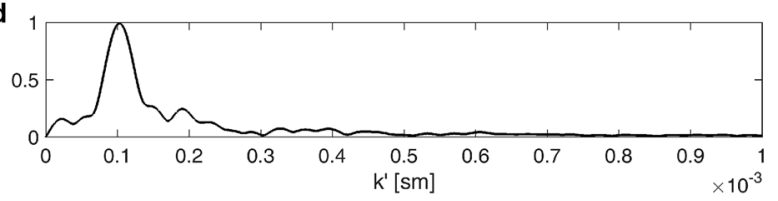

Fig. 4 Generic calculation of the oil-film thickness from the measurement data
The Fourier transforms of the functions $f\left(t^{-1}\right)$ and $g\left(t^{-1}\right)$ are known. $\mathcal{F}\left(f\left(t^{-1}\right)\right)$ is again a Gaussian function and $\mathcal{F}\left(g\left(t^{-1}\right)\right)$ yields

$$
\begin{aligned}
\mathcal{F}\left(\cos \left(2 \pi \frac{2 n_{\mathrm{eff}} C}{\lambda_{0}} t^{-1}\right)\right)(k) \\
=\frac{\delta\left(k-\frac{2 n_{\mathrm{eff}} C}{\lambda_{0}}\right)+\delta\left(k+\frac{2 n_{\mathrm{eff}} C}{\lambda_{0}}\right)}{2} .
\end{aligned}
$$

$\delta$ is the Delta function, which is only nonzero if its argument equals zero. Therefore $\mathcal{F}(f) * \mathcal{F}(g)$ yields two Gaussian functions symmetrical around zero with their maxima at $k= \pm 2 n_{\text {eff }} C / \lambda_{0}$.

The result of the Fourier transformed data of Fig. $4 \mathrm{~b}$ is shown in Fig. 4c. Due to the symmetry, only the positive side is shown and the Gaussian curves are normalized by their respective maxima. According to the difference in the mean wave lengths, the three Gaussian functions are shifted to each other. As a last step in the algorithm, the results are averaged by multiplying the individual curves by their respective mean wave length $\lambda_{0}\left(k^{\prime}=k \lambda_{0}\right)$. Thus the peak locations are at the same abscissa value. Then the values of the three curves were added up and the result divided by three. The averaged curve is shown in Fig. $4 \mathrm{~d}$, and the constant $C$ can be calculated by $C=k_{\text {peak }}^{\prime} /\left(2 n_{\text {eff }}\right)$.

Although $\theta_{\mathrm{i}}$ and $\mu_{\mathrm{oil}}$ are generally dependent on the surface coordinate of the model, in the present work both quantities are assumed to be constant. The calculated values of the oil-film thickness were inserted again into Eq. (2) to reconstruct the original interference pattern and validate the algorithm. The unknown parameters $I_{0}, I_{\mathrm{A}}$ and $L_{\mathrm{c}}$ in Eq. (2) were set by hand, and the result is shown in Fig. 5b. The interference image reconstructed on the basis of the pixelwise calculated oil-film thickness is in agreement with the original interference picture in Fig. 5a. The algorithm is even capable of reconstructing areas where the original interference pattern is disturbed due to obstacles in the flow. However, near the secondary separation line the reconstruction is less accurate. In this region, the steep slope of the oil-film thickness leads to large changes in the interference signal, which are averaged over the area covered by one pixel. This averaging leads to an indistinct interference oscillation and causes the reduced accuracy.

The wall streamline directions were also determined from the recorded interference pictures. The interference pattern contains longitudinal streaks from obstacles in the oil-film or an uneven oil-film thickness, which are aligned to the local flow direction (Schülein 2014). After careful analysis of the direction of the streaks, about 30 streamlines were traced manually with spline curves and then digitized. To reach a sufficient density of streamlines for wall shear stress calculations, additional streamlines were interpolated between 

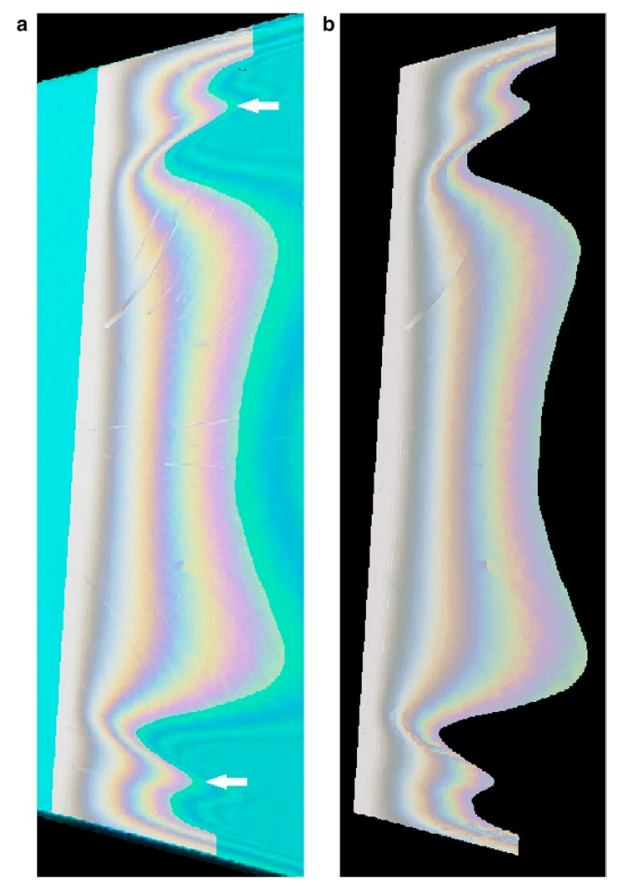

Fig. 5 Comparison between the original interference image (a) and the reconstructed image (b). Insufficient areas for the algorithm due to low interference intensities are masked light blue
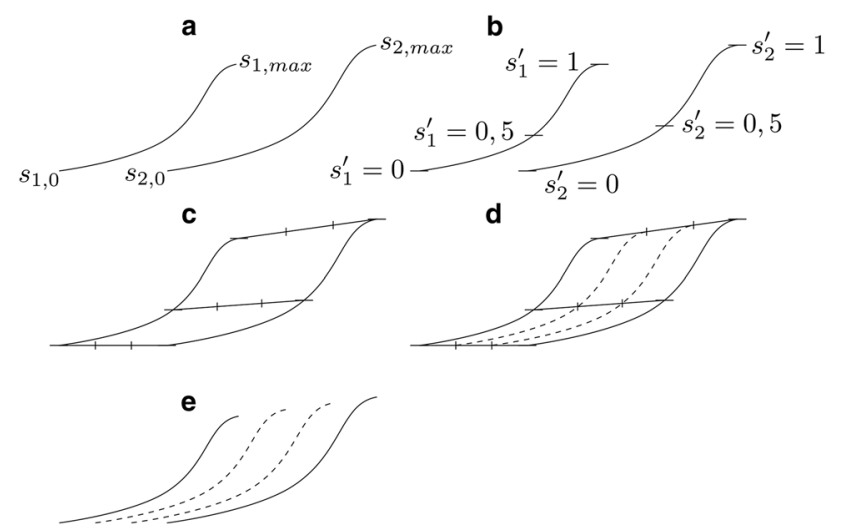

Fig. 6 Procedure for the interpolation of additional streamlines
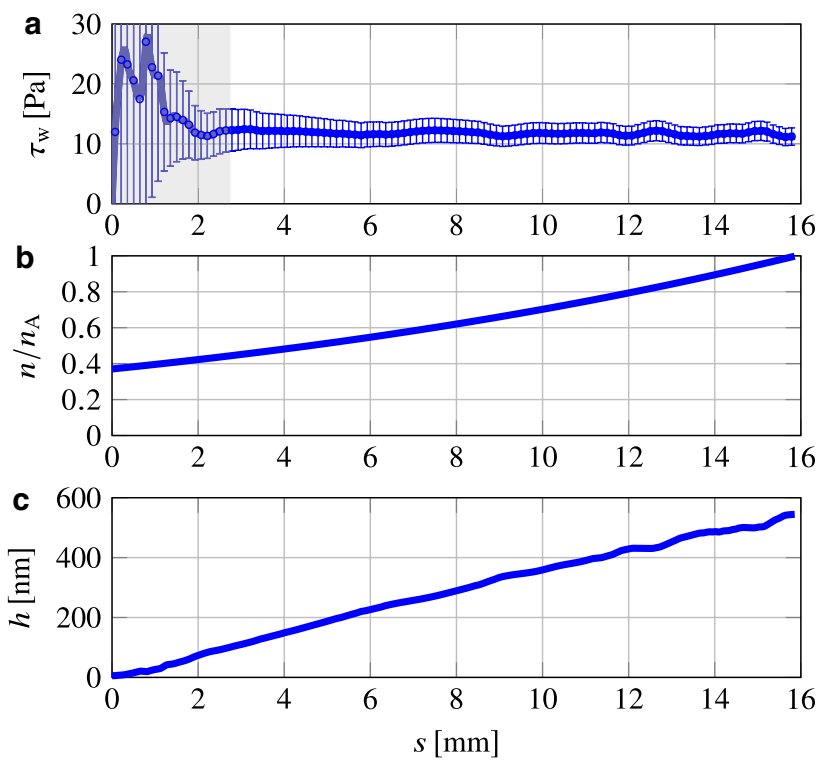

Fig. 8 Example for the calculation of the wall shear stress with errorbars along a wall streamline. The used oil-film thickness $h$ and width of the wall streamline are also shown. The discarded part of the wall shear stress for $h<100 \mathrm{~nm}$ is marked in gray

each two neighboring streamlines by a simple heuristic algorithm. The algorithm is based on the similarity of neighboring streamlines. After normalizing the digitized streamlines by their respective length $\left(s^{\prime}=s / s_{\max }\right)$, the characteristic points of the curves, like the inflection point, are at the same normalized coordinate. New streamlines can now be easily interpolated using the normalized coordinates of two known streamlines as support points. The individual steps of the interpolation are shown in Fig. 6 schematically. In the present work, 19 streamlines were interpolated between each two parent lines. One example is shown in Fig. 7. The local width $n$ of the oil stream tube is assumed to be given by the perpendicular distance between two neighboring streamlines.

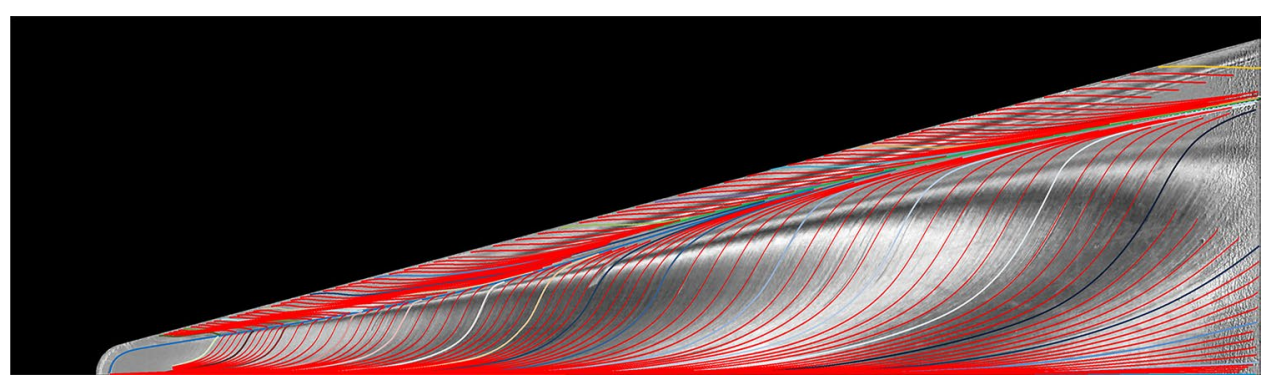

Fig. 7 A processed interference image for the determination of the wall streamline directions. The manually drawn wall streamlines follow the streaks in the interference pattern and are digitized using dis- tinct colors. The gaps between the hand-drawn lines are filled with interpolated streamlines using the proposed algorithm. Every fifth interpolated streamline is shown in the figure as a red line 


\section{Calculations of the wall shear stress}

After obtaining the oil-film thickness and the wall streamline direction from the interference images, the wall shear stress can be calculated with Eq. (1) along each streamline. Because the oil flows along the wall streamline direction, a smooth growth of the oil-film thickness can be expected. Therefore, an moving average of the oil-film thickness is calculated over approximately $0.5 \mathrm{~mm}$ (7 pixels). An example for the wall shear stress calculation is shown in Fig. 8a together with the used wall streamline width and smoothed oil-film thickness.

The wall shear stress shows a large variation close to the leading edge ( $s<2 \mathrm{~mm}$ ). Naughton and Hind (2013) reported about this problem in previous works and see the surface tension of the oil-film as the source for the large $\tau_{\mathrm{w}}$ variation. An other reason might be the used silicon oil (Böwing GmbH 2020). The silicon oil consists of polydimethylsiloxane, whose viscosity depends on the number of its monomer units. For a viscosity of $100 \mathrm{~mm}^{2} / \mathrm{s}$, the polydimethylsiloxane has approximately a molecular mass of $4700 \mathrm{u}$ (Kataoka and Ueda 1966) leading to 60 monomer units and roughly 600 atoms per molecule. A quick estimation about the size of the oil molecules by assuming that all atoms have a size of $1 \AA$ and order in a sphere, leads to silicon balls with a diameter of $0.8 \mathrm{~nm}$. Comparing the size of the silicon balls with the oil-film thickness close to the leading edge poses the question whether continuum mechanical approaches like the thin oil-film equation are still valid. Both effects lead to a systematic error in the determination of the oil-film thickness near the leading edge and cause the high $\tau_{\mathrm{w}}$ fluctuations. To avoid misinterpretations of the $\tau_{\mathrm{w}}$ distributions, all $\tau_{\mathrm{w}}$ values below $h<100 \mathrm{~nm}$ are discarded (cf. the gray box in Fig. 8a).

The errorbars of the wall shear stress are also shown in Fig. 8a and are calculated by Gaussian error propagation assuming a standard deviation of $\sigma_{n}=0.05 \cdot n, \sigma_{t}=1 \mathrm{~s}$, $\sigma_{\mu_{\mathrm{oil}}}=0.02 \cdot \mu_{\mathrm{oil}}$ and $\sigma_{h}=10 \mathrm{~nm}+0.04 \cdot h$. The standard deviation of $h$ has a systematic error of $10 \mathrm{~nm}$ due to the problems close to the leading edge. An analysis of the error propagation yields that the errorbars are dominated by the error of the oil-film thickness because Eq. (1) has a quadratic dependency of the local oil-film thickness $h_{\mathrm{A}}$. As a consequence, the errorbars decrease with the streamline distance $s$, because at higher $h$ the systematic error becomes irrelevant. For simplicity, the standard deviation of the wall shear stress in sufficiently high oil-films can be approximated by twice the relative error of $h\left(\sigma_{\tau_{\mathrm{w}}}=0.08 \cdot \tau_{\mathrm{w}}\right)$. The waviness of $\tau_{\mathrm{w}}$ in Fig. 8a is directly correlated with the local oil-film thickness, demonstrating the importance for measuring highly accurate oil-film thickness distributions. The accuracy of the current algorithm can be further improved by treating $\theta_{\mathrm{i}}$ as well as $\mu_{\mathrm{oil}}$ as location-dependent. Also, the acquisition rate of $0.2 \mathrm{~Hz}$ for the interference images is rather undersampled for the presented spectral analysis of the oil-film thickness. So an improved camera setup still holds a lot of potential for further increasing the accuracy of the wall shear stress measurement.

\section{Results and discussion}

The determined wall streamlines on the leeward side of the delta wing at an angle of attack of $\alpha=5^{\circ}$ are shown in Fig. 9 together with the magnitude of the calculated wall shear stress as a color plot. The separation and reattachment lines are marked in the figure and will be discussed in the following text.

The flow separates at the leading edge and rolls up into the primary vortex pair above the wing. At $\alpha=5^{\circ}$, the small size of the primary vortex leads to two distinct primary reattachment lines $R_{1}$ on the leeward side of the wing.
Fig. 9 Wall shear stress distribution and wall streamlines at $u_{\infty}=50 \mathrm{~m} / \mathrm{s}$ and $\alpha=5^{\circ}$

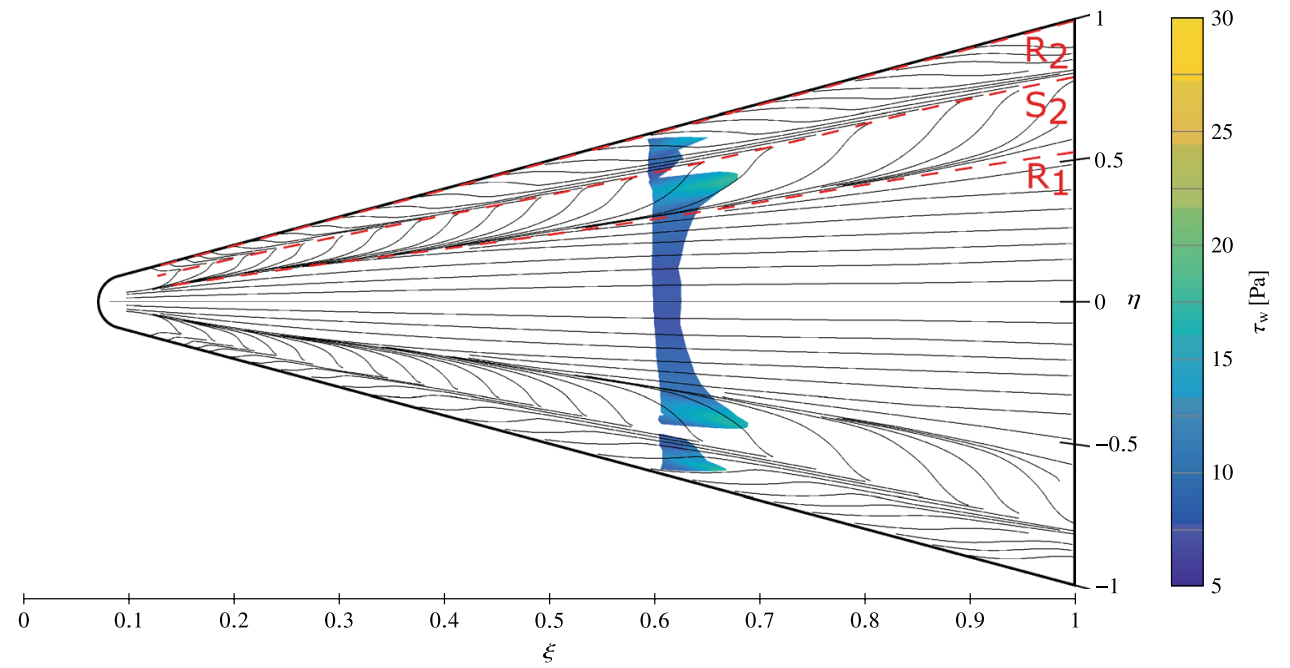



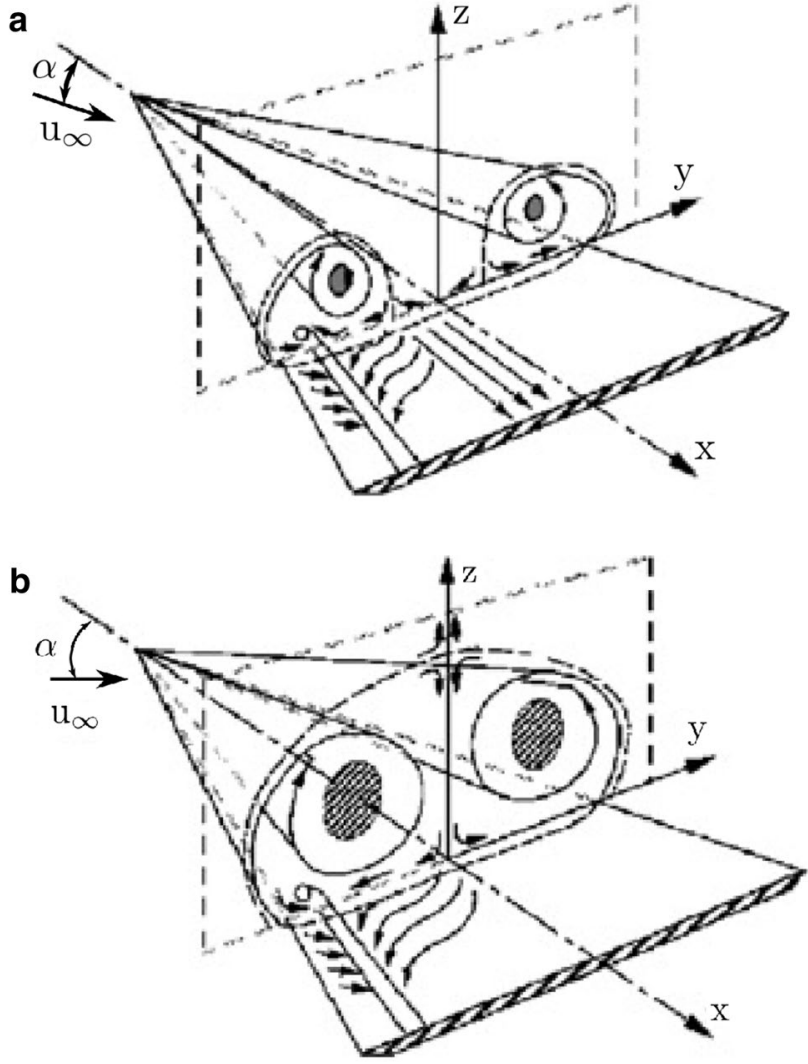

Fig. 10 Flow topology at the delta wing with two primary attachment lines (a) and one primary attachment line (b) (Breitsamter 2008)

A secondary flow forms from $R_{1}$ toward the leading edge due to the pressure minimum caused by the primary vortex. After passing the pressure minimum, the flow is slowed down through the positive pressure gradient and separates again at the secondary separation line $S_{2}$. The corresponding secondary reattachment line $R_{2}$ forms along the leading edge. The flow direction under the secondary vortex is from $R_{2}$ toward $S_{2}$.

Comparing this measured separation and reattachment lines with the flow topology described in other literature (Breitsamter 2008; Hummel 2004) shown in Fig. 10a, it is obvious that the flow structure deduced from the wall streamlines for $\alpha=5^{\circ}$ coincides.

In Fig. 9, the wall shear stress distribution is shown color-coded for a cross section at $\xi=0.62$. A maximum is visible beneath both primary vortex cores as a consequence of the vortex-induced flow acceleration at the wall. Under the secondary vortex, the same phenomenon occurs, indicated by a second peak. At the centerline of the wing, a small increase in the wall shear stress is barely visible in the color plot, but detectable in the quantitative values (cf. Fig. 14).

With increasing angle of attack, the size of the primary vortices also increases. This causes a shift of the primary reattachment lines $R_{1}$ and of the vortex axes toward the center of the wing. The increase in the size of the primary vortex is shown in Fig. 10 schematically. Figure 11 shows the experimental wall shear stress distribution and wall streamlines for $\alpha=15^{\circ}$. At this angle of attack, only a single primary reattachment line $R_{1}$ was detected at the centerline of the wing. The general level of wall shear stress is increased due to the higher near wall velocity gradients caused by the increased angle of attack and higher intensity of the primary vortices. Due to the enhanced size of the primary vortices, the location of the maximum wall shear stress is shifted toward the centerline compared to the $\alpha=5^{\circ}$ case.

In Fig. 12, the wall streamlines and wall shear stress distributions for $\alpha=25^{\circ}$ are shown. The most striking feature in the flow topology is the course of the secondary separation line. With increasing longitudinal coordinate between $0.4 \leq \xi \leq 0.55$, an unusual bending of the $S_{2}$ line

Fig. 11 Wall shear stress distribution and wall streamlines at $u_{\infty}=50 \mathrm{~m} / \mathrm{s}$ and $\alpha=15^{\circ}$

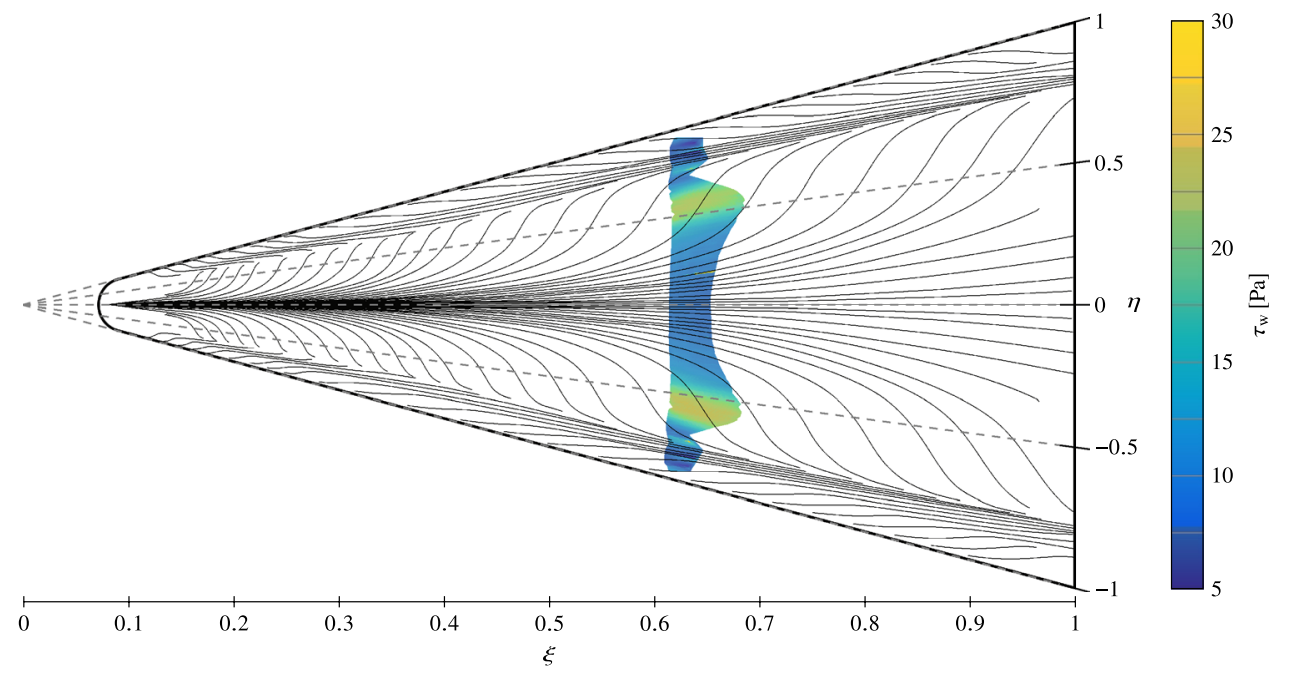


Fig. 12 Wall shear stress distribution and wall streamlines at $u_{\infty}=50 \mathrm{~m} / \mathrm{s}$ and $\alpha=25^{\circ}$

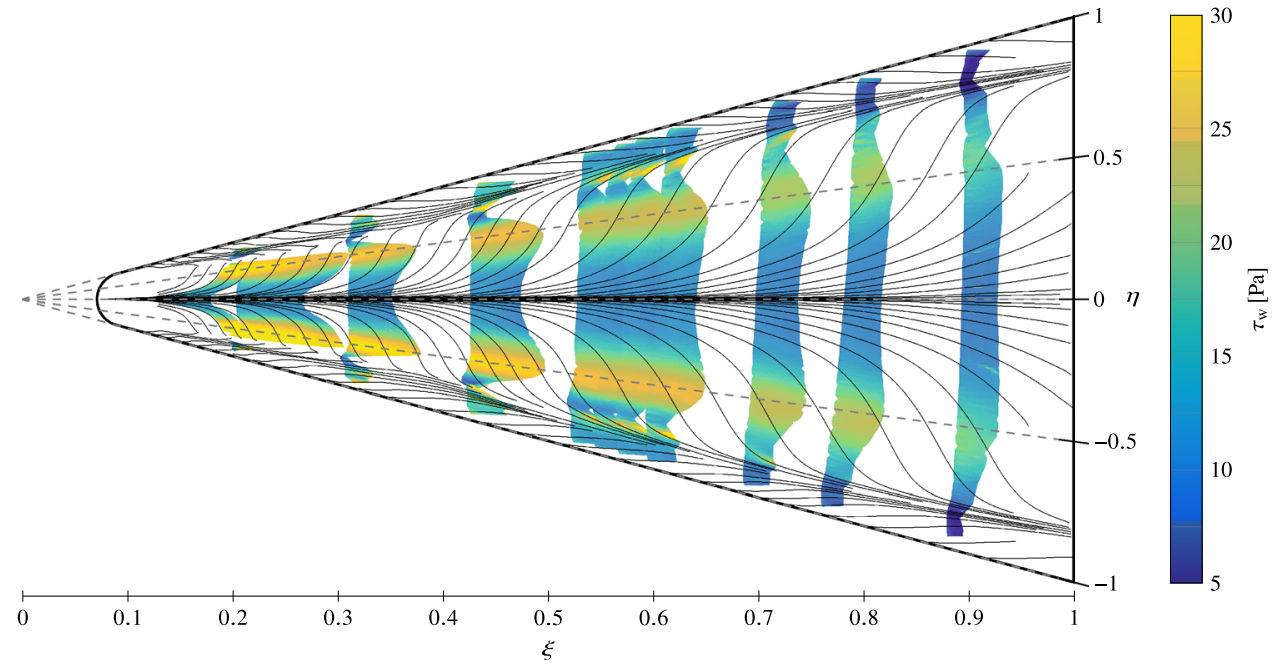

toward higher $\eta$ takes place. Upstream as well as downstream of the bending the secondary separation happens at a constant $\eta$ value, respectively, due to the conical symmetry of the model. This bending is also visible at smaller angles of attack. The explanation, which comes to mind first, is the change from a laminar boundary layer to a turbulent one. As well known, the turbulent boundary layer is more resistant to an adverse pressure gradient due to the higher kinetic energy near the wall, which may cause the delayed separation. The transition process is accompanied by an increased skin friction, but the measured WSS distributions show no transition induced rise. Hence, a transition between the laminar-turbulent boundary layer is rather unlikely as the cause of the bending.

An other explanation for the bending is based on the geometry of the investigated model. The generic delta wing has a rounded nose contour in the $\xi-\eta$-plane, which causes the generation of a horseshoe vortex (Délery 2013) due to leading edge separation at nonzero angles of attack. In contrast, at the main part of the wing the vortex structure is conical, consisting of a primary and secondary vortex pair (Fig. 10). This two different vortical structures have to merge somewhere over the leeward side of the wing. It is unclear at the moment where and how this transformation occurs, but in the transition region of this progress the pressure distribution is expected to change and maybe cause the bending of the $S_{2}$ line.

The wall shear stress was investigated more intensively along the wing for $\alpha=25^{\circ}$. The wall shear stress decreases along the conical rays (Fig. 12) according to the increase in the boundary layer thickness. Compared with $\alpha=5^{\circ}$ and $15^{\circ}$, the wall shear stress is further increasing for $\alpha=25^{\circ}$ at the same $\xi$ location because of the more intense primary vortex pair above the wing. The wall shear stress maximum on the left-hand side $(\eta<0)$ is slightly higher than on the righthand side $(\eta>0)$. The asymmetry in the calculated wall shear stress is the logical consequence of the asymmetry in the interference pattern in Fig. 5a. Despite the oblique leading edge of the oil-film, the interference pattern develops equally far. Such a lee-side-flow asymmetry is not unusual for experimental data and can be caused by the smallest asymmetry in both the mounting of the test model and the inflow conditions. At $\xi \approx 0.4-0.75$ the wall shear stress values drastically increase shortly upstream of the $S_{2}$ line and even surpass the maximum value under the primary vortex. These wall shear stress peaks are presumably associated with the bending of the $S_{2}$ line, because the sudden increase in the wall shear stress value can be first observed downstream of the $S_{2}$ bending and vanishes at the rear part of the wing. The high wall shear stress level can also be observed in the interference pattern (white arrows in Fig. 5a), because the interference fringes are broadly stretched despite the convergence of the wall streamlines.

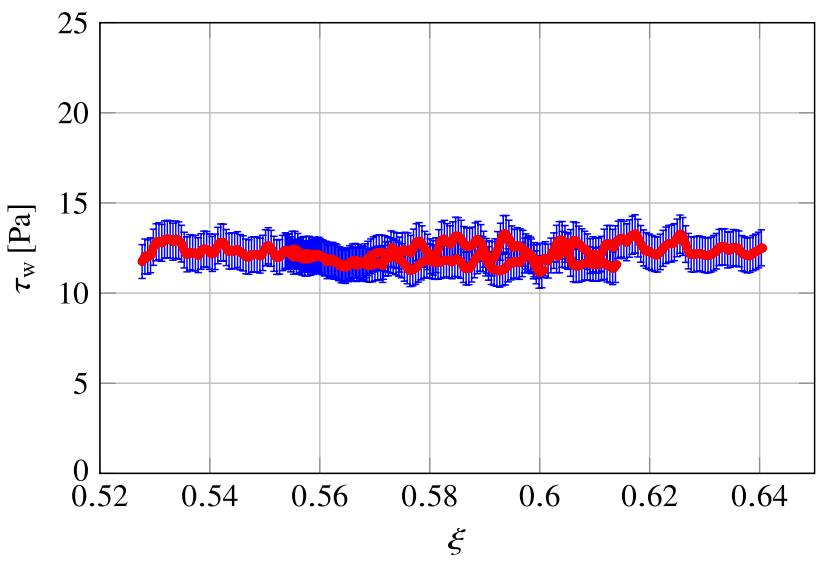

Fig. 13 Wall shear stress distributions with errorbars along the centerline of the wing at $\alpha=25^{\circ}$ between $\xi=0.53-0.64$. The wall shear stress distributions of the independent measurement runs overlap within the errorbars with each other 


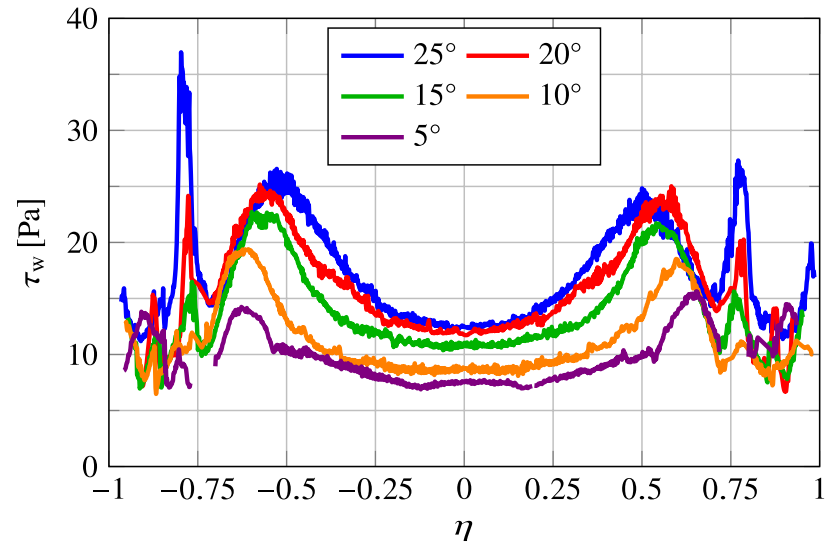

Fig. 14 Wall shear stress at different angles of attack at $\xi=0.62$

In the region of $\xi=0.53-0.64$, the measurements were repeated to verify the reproducibility of the calculation. Figure 13 shows that the calculated $\tau_{\mathrm{w}}$ distributions of the independent runs overlap with each other in regard to the errorbars. This consistency indicates that reproducibility is within the $8 \%$ measurement uncertainty.

For a better insight into the effects of the angle of attack on the wall shear stress, in Fig. 14 the experimental $\tau_{w}$ distributions for $\alpha=5^{\circ}-25^{\circ}$ are compared at the same longitudinal coordinate of $\xi=0.62$. A lot of effects discussed above become more pronounced due to this comparison. The major findings are the increase in the overall wall shear stress level including the wall shear stress maximum as well as the shift of the maximum locations toward the centerline with increasing angle of attack.

In the measurements discussed above, the oil was applied across the longitudinal axis of the delta wing. The benefit of this technique lies in the ability to capture regions with separation and reattachment lines equally well, but its drawback is that only a small size of the test area is available for evaluation after each single wind-tunnel run. Alternatively, the oil can also be applied along the centerline of the wing. With this approach, a larger area can be measured simultaneously at a single run, like it is shown in Fig. 15. However, the analysis is limited in this case by the location of the secondary separation lines, since they are forming an obstacle for the oil stream. The evaluated wall shear stress distribution changes suddenly in Fig. 15 at a few locations (see the red arrows in the color plot). The analysis of the wall shear stress distribution happens in segments between limiting streamlines. Therefore, these jumps in the wall shear stress distribution result from flaws in the determination of the limiting streamlines.

The calculated wall shear stress distributions for the cross section at $\xi=0.62$ are shown for both methods of oil application in Fig. 16. Because the discrepancies are on average under 5\% and therefore within the measurement accuracy, both techniques yield equivalent results and can be used for wall shear stress measurement depending on the elucidated pros and cons.

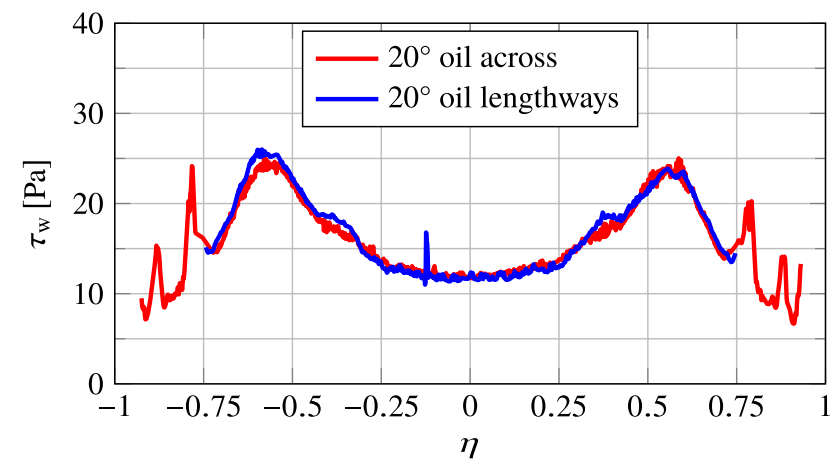

Fig. 16 Comparison of the wall shear stress profiles at $\xi=0.62$ of the two different methods
Fig. 15 Wall shear stress distribution and wall streamlines at $u_{\infty}=50 \mathrm{~m} / \mathrm{s}$ and $\alpha=20^{\circ}$. The oil was applied along the centerline of the wing

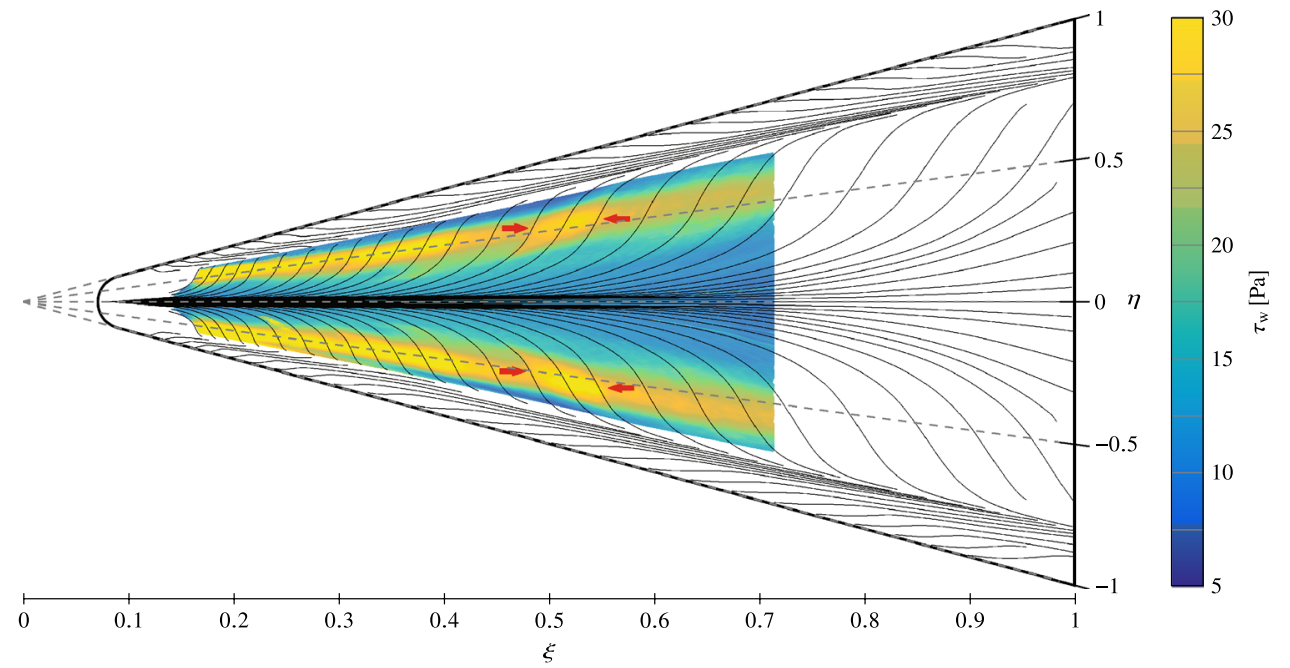




\section{Conclusion}

The capabilities of the oil-film interferometry with white light to determine the flow topology and the wall shear stress distribution were demonstrated on a generic delta wing model with a cost efficient setup. The analysis of the interference images was simplified by a physically based algorithm. It enables the automatic calculation of the oilfilm thickness distribution from a sequence of interference images. A reconstruction of the measured interference patterns was conducted for validation and demonstrates the quality of the algorithm. Critical points in the calculation of the oil-film thickness were discussed, and steps for further improvements were outlined.

The flow topology, which was deduced from the wall streamlines visible in the interference images, is consistent with established data. Merely the bending of the $S_{2}$ line could not be found in the literature. It was inferred that the bending is likely caused by the interaction of the horseshoe vortex generated at the rounded nose of the test model with the main vortical structures emerging over the delta wing. Although the proposed wall streamline interpolation method simplifies the analysis of the interference images, the determination of the wall streamline direction is not objective and still time-consuming. A next step for the oil-film interferometry lies in an objective and reliable method for the wall streamline direction.

The wall shear stress distributions were presented and analysed for selected angles of attack. The measurement uncertainty was estimated by the Gaussian error propagation leading to a standard deviation of $8 \%$. The wall shear stress distributions are plausible with the flow topology as well as with the interference images. Various known features in the wall shear stress distributions were distinguishable in the color plots, like the increased wall shear stress under the primary vortex or the effect of the boundary layer thickness on the wall shear stress. The quantitative wall shear stress distributions were used to describe the primary vortex intensity and kernel position for different angles of attack.

To investigate the applicability of the implemented data reduction methods two different approaches of oil-film application to the model surface were tested at a fixed flow condition. A comparison of the determined $\tau_{w}$ values yield an averaged deviation below 5\%, which is within the measurement uncertainty. Therefore, both techniques were recognized to be suited for the wall shear stress measurements in 3-D flows confirming the reliability of the proposed algorithms for the quantitative analysis of flow effects.

Acknowledgements Open Access funding provided by Projekt DEAL. This study was partially funded by the German-Dutch Wind Tunnels, DNW, in the scope of the project Evaluation of Image Based SkinFriction Diagnostics for Application in Subsonic Flows.
Open Access This article is licensed under a Creative Commons Attribution 4.0 International License, which permits use, sharing, adaptation, distribution and reproduction in any medium or format, as long as you give appropriate credit to the original author(s) and the source, provide a link to the Creative Commons licence, and indicate if changes were made. The images or other third party material in this article are included in the article's Creative Commons licence, unless indicated otherwise in a credit line to the material. If material is not included in the article's Creative Commons licence and your intended use is not permitted by statutory regulation or exceeds the permitted use, you will need to obtain permission directly from the copyright holder. To view a copy of this licence, visit http://creativecommons.org/licenses/by/4.0/.

\section{References}

Baldwin A, Mears LJ, Arora N, Kumar R, Alvi FS, Naughton JW (2019) Skin friction measurements using oil film interferometry in a 3-d supersonic flowfield. AIAA J 57(4):1373-1382

Böwing GmbH L (2020) Technical information ELBESIL-ÖLE B

Breitsamter C (2008) Unsteady flow phenomena associated with leading-edge vortices. Prog Aerosp Sci 44:48-65

Brown JL, Naughton JW (1999) The thin oil film equation. NASA/ TM-1999-208767

Délery J (2013) Three-dimensional separated flow topology. Wiley, New Jersey

Desse JM (2003) Oil-film interferometry skin-friction measurement under white light. AIAA J 41(12):2468-2477

Driver DM (1997) Application of oil film interferometry skin-friction to large wind tunnels. In: AGARD conference proceedings CP 201, Paper 25, advanced aerodynamic measurement technology, Seattle, WA, USA

Driver DM, Drake A (2008) Skin friction measurements using oilfilm interferometry in nasa's 11 -foot transonic wind tunnel. AIAA J 46(10):2401-2407

Garrison TJ, Ackman M (1998) Development of a global interferometer skin-friction meter. AIAA J 36(1):62-68

Haritonidis JH (1989) The measurement of wall shear stress. Springer, Berlin, pp 229-261

Hummel D (2004) Effects of boundary layer formation on the vortical flow above slender delta wings. RTO AVT symposium on "Enhancement of NATO military flight vehicle performance by management of interacting boundary layer transition and separation"

Kataoka T, Ueda S (1966) Viscosity-molecular weight relationship for polydimethylsiloxane. J Polym Sci Part B Polym Lett 4(5):317-322

Kim KS, Settles GS (1990) Skin friction measurements by laser interferometry in swept shock/boundary-layer interactions. AIAA J 28(1):133-139

Lunte J (2016) Optical wall shear stress measurement in threedimensional flows through white light interferometry. Master's thesis, Uni Göttingen, DLR Göttingen. (in German)

Lunte J, Schnepf C, Schülein E (2018) Optical wall shear stress measurements on the leeward side of a delta wing. 2018 Aerodynamic measurement technology and ground testing conference, AIAA 2018-3806

Lunte J, Schülein E (2018) Skin friction measurements in threedimensional flows by white-light oil-film interferometry. In: New results in numerical and experimental fluid mechanics XI, Springer, New York, pp 567-576

Maksimov AI, Pavlov A, Shevchenko AM (1994) Development of the optical skin friction measurement technique for supersonic gradient flows. In: Proceedings of the international conference on the methods of aerophysical research, pp 172-177 
Mateer GG, Monson DJ, Menter FR (1996) Skin-friction measurements and calculations on a lifting airfoil. AIAA J 34(2):231-236

Monson DJ, Mateer GG, Menter F (1993) Boundary-layer transition and global skin-friction measurement with an oil-fringe imaging technique. SAE Paper No. 932550

Monson DJ (1983) A nonintrusive laser interferometer method for measurement of skin friction. Exp Fluids 1(1):15-22

Monson DJ (1984) A laser interferometer for measuring skin friction in three-dimensional flows. AIAA J 22(4):557-559

Monson DJ, Higuchi H (1981) Skin friction measurements by a duallaser-beam interferometer technique. AIAA J 19(6):739-744

Naughton JW, Brown JL (1996) Surface interferometric skin-friction measurement technique. In: Advanced measurement and ground testing conference, $\mathrm{p} 2183$

Naughton JW, Brown JL (1997) Skin-friction distribution near a cylinder mounted on a flat plate. AIAA paper 97-1783

Naughton JW, Hind MD (2013) Multi-image oil-film interferometry skin friction measurements. Meas Sci Technol 24(12):124003

Naughton JW, Sheplak M (2002) Modern developments in shear-stress measurement. Prog Aerosp Sci 38:515-570

Schülein E (2006) Skin friction and heat flux measurements in shock/ boundary layer interaction flows. AIAA J 44(8):1732-1741

Schülein E (2014) Optical method for skin-friction measurements on fast-rotating blades. Exp Fluids 55(2):1672

Settles G (1986) Recent skin friction techniques for compressible flows. In: 4th joint fluid mechanics, plasma dynamics and lasers conference, $\mathrm{p} 1099$
Siller HA, Perkins RJ, Janke G (1992) Image analysis of oil film interferometry-a method of measuring wall shear stress distributions. Appl Sci Res 49(3):261-270

Squire LC (1962) The motion of a thin oil sheet under the steady boundary layer on a body. In: Maltby RL (ed) Flow visualization in wind tunnels using indicators, AGARDograph 70

Tanner LH (1977) A skin friction meter, using the viscosity balance principle, suitable for use with flat or curved metal surfaces (based on thickness measurement). J Phys E Sci Instrum 10(3):278

Tanner LH, Blows LG (1976) A study of the motion of oil films on surfaces in air flow, with application to the measurement of skin friction. J Phys E Sci Instrum 9(3):194

Tanner L, Kulkarni V (1976) The viscosity balance method of skin friction measurement: further developments including applications to three-dimensional flow. J Phys E Sci Instrum 9(12):1114

Winter K (1979) An outline of the techniques available for the measurement of skin friction in turbulent boundary layers. Prog Aerosp Sci 18:1-57

Zilliac GG (1998) Skin friction distribution on a wingtip. AIAA Paper 98-0584

Publisher's Note Springer Nature remains neutral with regard to jurisdictional claims in published maps and institutional affiliations. 